\title{
Transcriptomic mechanisms of Solanum tuberosum defensive response to golden potato nematode infestation
}

\author{
Alexey Kochetov \\ ICG SB RAS, Novosibirsk, Russia \\ ak@bionet.nsc.ru \\ Kseniya Strygina \\ VIR, st. Petersburg, Russia \\ pushpandjali@gmail.com \\ Elena Khlestkina \\ VIR, st. Petersburg, Russia \\ director@vir.nw.ru
}

\author{
Egorova Anastasiya \\ ICG SB RAS, Novosibirsk, Russia \\ egorova@bionet.nsc.ru \\ Dmitry Afonnikov \\ Kurchatov Genomics Center, \\ ICG SB RAS, Novosibirsk, Russia \\ ada@bionet.nsc.ru \\ Sophia Gerasimova \\ Kurchatov Genomics Center, \\ ICG SB RAS, Novosibirsk, Russia \\ gerasimova@bionet.nsc.ru
}

\author{
Anastasiya Glagoleva \\ Kurchatov Genomics Center, \\ ICG SB RAS, Novosibirsk, Russia \\ glagoleva@bionet.nsc.ru \\ Nickolay Shmakov \\ ICG SB RAS, Novosibirsk, Russia \\ shmakov@bionet.nsc.ru
}

\begin{abstract}
Golden potato cyst nematode (GPCN) Globodera rostochiensis is one of the major potato pathogens, and has a sophisticated mechanism of interaction with roots of the host plants. Resistance of cultivated $S$. tuberosum forms to GPCN infestation is commonly based on specific $R$ genes introgressed from natural populations of related varieties. In this research, RNA-seq is implemented in order to investigate $R$ genes composition of two $S$. tuberosum varieties with contrasting resistance to GPCN, and transcriptomic response to infestation is studied.
\end{abstract}

Keywords - transcriptomics, RNA-seq, potato, nematode, defensive response

\section{Motivation and Aim}

\section{Motivation}

S. tuberosum is a major crop cultivated worldwide and providing a substantial amount of calories in both human food and cattle feed. GPCN is a quarantine pest found responsible for yield losses that can range from $19 \%$ to $90 \%$, depending on potato cultivar. Protection of plants against potato cyst nematodes commonly involves the usage of nematicides or trap crops. However, many chemical nematicides are either toxic or have low efficiency.

Alternatively, introduction of $\mathrm{R}$ genes associated with nematode resistance is used to protect crops. Main source of such genes are natural populations of Solanum species. For example, $\mathrm{R}$ genes conferring strong resistance to the pathotype Ro1 of GPCN were introgressed from South America originated species: the H1 gene from the Solanum tuberosum subsp. andigenum and Gro1-4 gene from the Bolivian species $S$. spegazzinii.

In Russia, GPCN occurs locally in some regions of the European part, southern Siberia, and the Far East. Coupled with importance and wide usage of potato in agriculture in Russian Federation, this facilitates the search for new genes associated with GPCN resistance of $S$. tuberosum, which makes creation of new resistant varieties possible.

Aim

This research aims to investigate transcriptomic response of two $S$. tuberosum cultivars to GPCN infestation. The cultivars used in this study are contrasting in resistance to GPCN. Resistance to nematode infestation is mediated through HR-response and programmed cell death that is commonly connected to NBS-LRR type receptors [1] Thus, one of the main objectives of such transcriptome investigation was to identify R genes, and more specifically, NBS-LRR genes, associated with GPCN resistance specific to the resistant line for further investigation and introgression in potato through conventional breeding or other biotechnological approaches. At the same time, sets of genes differentially expressed between two cultivars at different time stages and after inoculation with GPCN cysts and control treatment with water were identified, and functional annotation of DEGs was performed in order to study general transcriptomic response of $S$. tuberosum to nematode infestation.

\section{Methods}

In order to study specifics of plant-nematode interaction and defense mechanisms, two accessions of $S$. tuberosum - k11291 (collected in Peru) and k-9836 (from Bolivia) - were selected. Accession k-9836 is susceptible to GPCN, while k11291 is highly resistant, but contains no DNA markers of Gro1-4 and H1, commonly associated with resistance to the nematode. RNA-seq was performed in order to study transcriptome of the two plant cultivars. Total RNA was collected collected before inoculation ( 0 hours), 24 and 72 hours after inoculation with $G$. rostochiensis (pathotype Ro1). For each genotype, three infected and three control (waterinoculated) plants were taken, which gives us a total of 30 mRNA samples. Illumina NextSeq 550 platform was used for sequencing of $2 \times 150$ reads.

After libraries filtering, short reads were aligned to the $S$. tuberosum reference genome using STAR maps. Differential expression search was performed with EdgeR package, and Gene Ontology terms enrichment analysis was carried out.

Additionally, de novo transcriptome assembly was performed using Trinity assembler, and obtained transcriptomes were searched for R genes through NBS and LRR domain prediction. Assembled transcriptome was aligned to the reference genome with aid of BLAT aligner in order to identify novel transcript. hmmscan utility from HMMER package was implemented for domain structure prediction of transcripts, and NLR-parser software was used to specifically identify transcripts containing NBS and LRR domains associated with plant immunity and defense responses. 
For a set of specific genes of interest, qRT-PCR expression quantification was performed in order to validate predicted differential expression.

\section{Results}

Functional annotation of DEGs revealed a strong difference in transcriptomes dynamics in the roots of resistant and susceptible $S$. tuberosum varieties in response to nematode infestation. While susceptible variety's response to GPCN cysts inoculation was similar to the response to simple water treatment performed as a control, and included activation of genes associated with cell cycle and mitosis, resistant variety's response to GPCN was quite different and included up-regulation of genes associated with stress response, and activation of peptidase inhibitor- and glycosylase-encoding genes.

De novo transcriptome assembly revealed a number of transcripts with no annotation in reference transcriptome that are actively expressing in the resistant line. Namely, a contig was identified that has no homology to the annotated genome sequence of $S$. tuberosum nor has any presence in the transcriptome of the susceptible potato variety, and at the same time has NBS and LRR domains. This contig is a promising molecular marker of nematode resistance and can further be implemented in engineering of new agriculturally important varieties of potato.

\section{Acknowledgment}

This research is supported by RSF grant 16-16-04073. Plants were cultivated in the IC\&G greenhouse facility (supported by budget project 0324-2016-0001)

\section{References}

[1] Kochetov AV, Glagoleva AY, Strygina KV, Khlestkina EK, Gerasimova SV, Ibragimova SM, Shatskaya NV, Vasilyev GV, Afonnikov DA, Shmakov NA, Antonova OY, Gavrilenko TA, Alpatyeva NV, Khiutti A, Afanasenko OS. Differential expression of NBS-LRR-encoding genes in the root transcriptomes of two Solanum phureja genotypes with contrasting resistance to Globodera rostochiensis. BMC Plant Biol. 2017;17(Suppl 2):251 